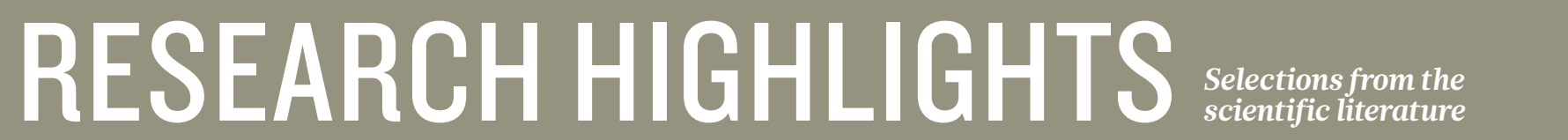

VIROLOGY

\section{Improved mouse for Ebola research}

To overcome the lack of good mouse models for Ebola studies, researchers have created a mouse that displays the hallmark symptoms of Ebola infection - potentially useful for drug and vaccine development.

A team led by Michael Katze at the University of Washington, Seattle, crossed eight strains of mice to produce a colony with a diverse genetic background. When infected with a mouse-adapted version of the Ebola virus, the mice exhibited a broad range of responses from no observable symptoms to severe haemorrhagic fever.

Surviving mice showed key physiological differences compared with susceptible animals, such as immune cells that function better. The researchers link these differences to variants of the genes Tie 1 and Tek.

Science http://doi.org/ws4 (2014)

\section{PHYSIOLOGY}

\section{How baby bones self-repair}

Infant bone fractures heal without any medical intervention, thanks to muscle contractions and

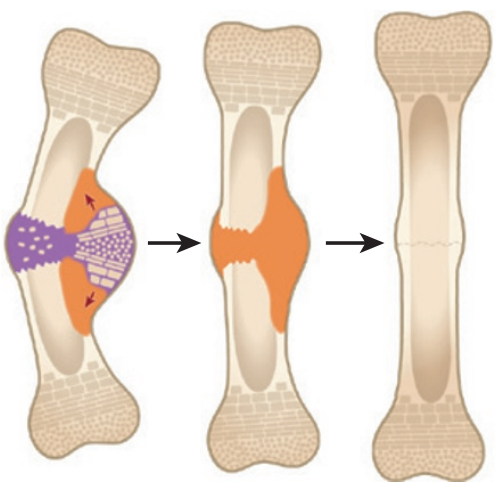

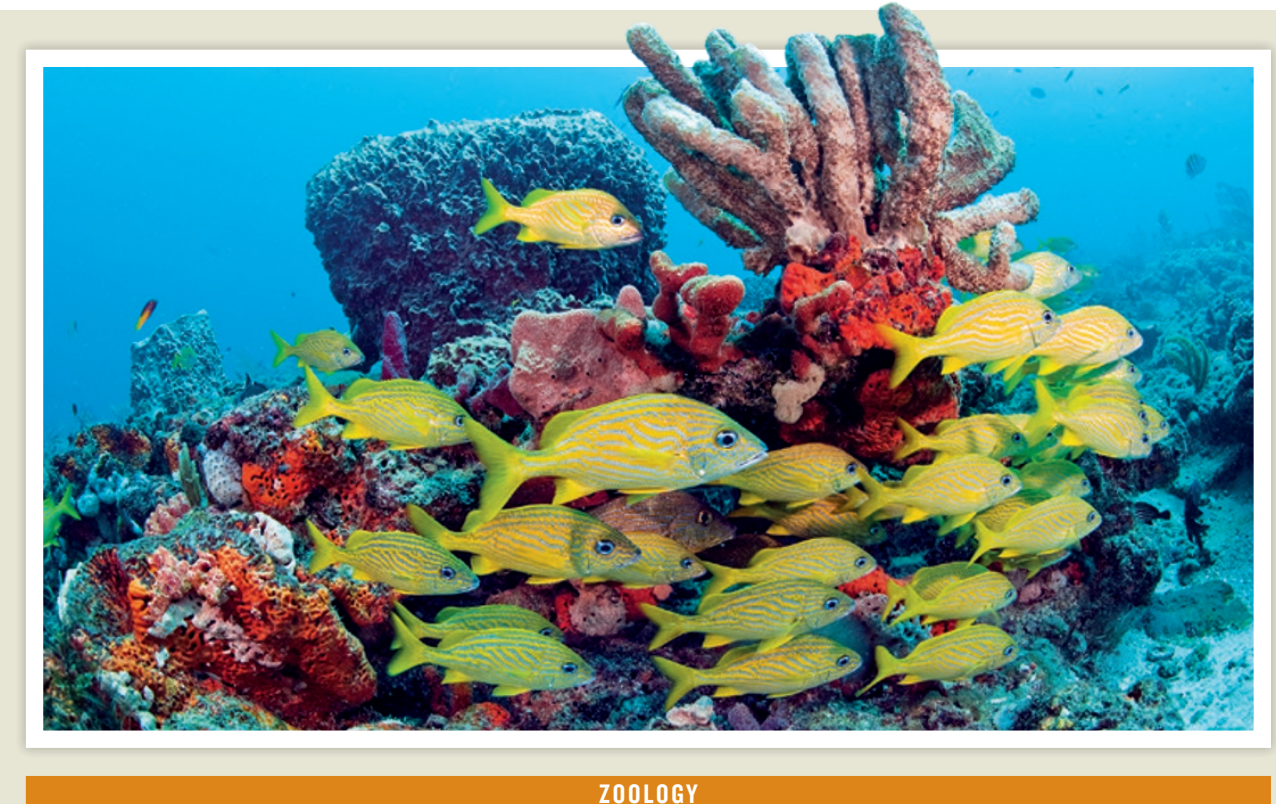

\section{Fish grind teeth to grunt}

A species of fish makes grunting sounds by grinding its teeth. The French grunt fish (Haemulon flavolineatum; pictured) is thought to groan in distress by rubbing together its pharyngeal jaws, a second pair of toothed jaws located in the throat that help to process food. Frédéric Bertucci of the University of Liége in Belgium and his co-workers used X-ray cameras to capture high-speed video of the animals and found that the sound-making process was similar to chewing, but slightly slower. Electron microscope images of the teeth revealed signs of erosion.

The fish's hearing is not tuned specifically to the frequencies of the grunts, suggesting that the pharyngeal jaws first evolved for eating and were later co-opted for communication.

J. Exp. Biol. 217, 3862-3869 (2014) tissue growth that together move the bone fragments back into place.

Elazar Zelzer and his colleagues at the Weizmann Institute of Science in Rehovot, Israel, imaged fractured bones as they healed in newborn mice. They found that a soft callus (pictured in purple) containing cartilage forms at the fracture site and acts like a mechanical jack: the growth of tissue (red arrows) on one side of the fracture provides force to realign the bone fragments. The callus hardens (orange), allowing the bone to heal.

Botulinum toxin, which paralyses muscles, blocked the repair process in the mice, suggesting that muscle contractions are also required for infant bone healing. Dev. Cell 31, 159-170 (2014)

\section{MATERIALS \\ Device bends light from all angles}

A material can distort the refraction of infrared light from almost any angle, paving the way for new kinds of optical device.

Such metamaterials are made up of tiny structures that tune electromagnetic waves in ways that would be impossible in a natural material. But they work only for long wavelengths, or with light coming from certain directions.

To create the structure, Takuo Tanaka at the RIKEN Metamaterials Laboratory in Saitama, Japan, and his colleagues first built a flat template out of an etched polymer, silicon and metal strips. When exposed to air, stresses in the strips caused them to curl into threedimensional rings, a structure that unnaturally bent light coming from almost any angle.

The authors say that the approach could eventually be used in devices such as superlenses, which allow scientists to see beyond the limit of conventional lenses. Adv. Opt. Mater. http://doi.org/ ws3 (2014) 